\title{
BOUNDING THEOREM IN FINITE PLASTICITY WITH HARDENING EFFECT*
}

\author{
BY \\ YANG GAO ${ }^{1}$ AND TOMASZ WIERZBICKI \\ Massachusetts Institute of Technology, Cambridge, MA
}

\begin{abstract}
The complementary variational problems for finite plasticity with strainhardening effect are developed in this paper based on the theory of convex analysis. A new lower bound theorem for the load intensity factor, in which the plastic yield condition is relaxed by a complementary plastic superpotential, is proved. A simple illustrative example is worked out.
\end{abstract}

1. Introduction. Complementary variational techniques for deriving approximate solutions to initial-boundary value problems have received considerable interest in recent years [1,2]. Based on the theory of nonsmooth analysis, a systematic treatment of geometrically linear systems of elastoplasticity was presented in [3]. It was shown that there exists a full symmetry between the primal and complementary variational problems. But, in the presence of geometrical nonlinearity, a number of fundamental questions related to the complementary energy principle for nonlinear mechanics still remain obscure.

Recently, the complementary principles for nonlinear variational boundary-initial value problems have been studied in [4]. By introducing the so-called complementary gap function, an interesting symmetry between the primal and dual variational problems was proved with some important implications in nonlinear mechanics. The term "gap" indicates that there is a difference between the conjugate function of the potential energy functional and the complementary functional in the geometrically nonlinear cases. Similar functions to the present gap function were considered earlier by Onat [5] and Wierzbicki and Ploch [6] in the analysis of uniqueness and bounding theorems in finite plasticity for one-dimensional problems. In the present paper, an application of the gap function is given to problems of finite plasticity. A new bound theorem is constructed, in which the plastic yield condition is relaxed by the complementary plastic superpotential. It is proved that the existence of the bound is closely related to the property of the gap function.

${ }^{*}$ Received June 15, 1988.

' Permanent address: Department of Applied Mathematics and Mechanics, Hefei Polytechnic University, Hefei, P. R. China.

(C)1989 Brown University 
2. Constitutive relation and governing equations. Let $\Omega$ be an open, bounded, connected subset of $\mathbf{R}^{3}$ with Lipschitz boundary $\Gamma, \mathbf{U}$ and $\boldsymbol{\Sigma}$ are the admissible velocity space and Kirchhoff stress space, respectively; $A: \mathbf{U} \rightarrow \mathbf{E}$ is a nonlinear geometric operator:

$$
A v:=\frac{1}{2}[\nabla v+v \nabla+(\nabla v)(v \nabla)]=\epsilon(v) \in \mathbf{E} .
$$

$\mathbf{E}$ is the conjugate space of $\boldsymbol{\Sigma}$, i.e., the admissible Green strain space. The directional derivative of $\epsilon$ at $u$ in the direction $v \in \mathbf{U}$ is defined as

$$
\delta \epsilon(u ; v):=\lim _{t \rightarrow 0^{+}} \frac{\epsilon(u+t v)-\epsilon(u)}{t}=A_{1}(u) v
$$

where $A_{1}: \mathbf{U} \rightarrow \mathbf{E}$ is called the Gâteaux derivative, [4,7] of $\epsilon(u)$ at $u$, which is also a nonlinear geometric mapping:

$$
A_{1}(u) v=\frac{1}{2}[\nabla v+v \nabla+(\nabla v)(u \nabla)+(\nabla u)(v \nabla)] .
$$

So the material derivative of $\epsilon$ may be written as

$$
\dot{\epsilon}(u)=A_{1}(u) \dot{u} \in \dot{\mathbf{E}}
$$

where $\dot{\mathbf{E}}$ is the admissible strain rate space. The conjugate operator $A_{1}^{*}: \boldsymbol{\Sigma} \rightarrow \mathbf{L}(\mathbf{L}$ is admissible force space) may be given by Gaussian transformation:

$$
\left\langle A_{1}(u) \dot{u}, S\right\rangle_{\Omega}=\left\langle\dot{u}, A_{1}^{*}(u) S\right\rangle_{\bar{\Omega}} .
$$

The bilinear form $\langle *, *\rangle: \dot{\mathbf{E}} \times \mathbf{\Sigma} \rightarrow \mathbf{R}$ is defined as $\langle\dot{\epsilon}, S\rangle=\dot{\epsilon}_{i j} S_{i j}$, and $\langle *, *\rangle_{\Omega}:=$ $\int_{\Omega}\langle *, *\rangle d \Omega,\langle *, *\rangle_{\Omega}:=\int_{\Omega}\langle *, *\rangle d \Omega+\int_{\Gamma}\langle *, *\rangle d \Gamma$. It is interesting that $A_{1}^{*}$ is just the equilibrium operator [4]:

$$
A_{1}^{*}(u) S= \begin{cases}-[(I+\nabla u) S] \cdot \nabla & \text { in } \Omega \\ (I+\nabla u) S \cdot n & \text { on } \Gamma .\end{cases}
$$

For rigid-hardening plastic media, the constitutive equation may be written in subdifferential inclusion form [3]:

$$
(\dot{\epsilon},-\dot{\vartheta}) \in \partial W^{*}\left(S, \vartheta^{*}\right),
$$

where $\vartheta \in \Theta$ is an internal variable. For example, $\vartheta$ can be defined as a dimensionless plastic work:

$$
\vartheta=\frac{1}{\Omega \sigma_{b}} \int_{0}^{t} d t \int_{\Omega} S \dot{\epsilon} d \Omega
$$

Certainly, other definitions might also be useful. $\vartheta^{*} \in \Theta^{*}$ is the conjugate variable of $\vartheta$, and the plastic superpotential $W^{*}: \Sigma \times \boldsymbol{\Theta}^{*} \rightarrow \tilde{\mathbf{R}}=\mathbf{R} \cup\{+\infty\}$ is defined as

$$
W^{*}\left(S, \vartheta^{*}\right)= \begin{cases}0 & \text { if }\left(S, \vartheta^{*}\right) \in \mathbf{K} \\ +\infty & \text { otherwise }\end{cases}
$$

where $\mathbf{K} \subset \boldsymbol{\Sigma} \times \boldsymbol{\Theta}^{*}$ is a convex subset:

$$
\mathbf{K}:=\left\{\left(S, \vartheta^{*}\right) \in \mathbf{\Sigma} \times \mathbf{\Theta}^{*} \mid f\left(S, \vartheta^{*}\right)=J(S)-\sigma_{b}-F\left(\vartheta^{*}\right) \leq 0 \text { in } \Omega\right\},
$$

in which $J(S)$ is a convex function of the stress tensor $S, \sigma_{b}$ is a material constant, $F\left(\vartheta^{*}\right)$ is a hardening function. The concept of superpotential was first proposed by 
Moreau [13] in the surface friction problem. We introduce it here to emphasize that the function $W^{*}$ may not be continuous. In the theory of convex analysis (cf. e.g. [7]), $W^{*}$ is called the indicator of the convex set $\mathbf{K}$; it is convex, lower semicontinuous, and subdifferentiable. $\partial W^{*}\left(S, \vartheta^{*}\right)$ denotes the subdifferential of $W^{*}$ at $\left(S, \vartheta^{*}\right)$, which is a convex subset of $\mathbf{E} \times \Theta[3]$ :

$$
\partial W^{*}(S):= \begin{cases}\dot{\lambda} \partial f\left(S, \vartheta^{*}\right) & \text { if } f\left(S, \vartheta^{*}\right)=0, \dot{\lambda} \geq 0, \\ \{0\} & \text { if } f\left(S, \vartheta^{*}\right)<0, \\ \varnothing & \text { if } f\left(S, \vartheta^{*}\right)>0 .\end{cases}
$$

I.e., under the constraint $f\left(S, \vartheta^{*}\right)=0, \dot{\lambda} \geq 0$, the constitutive relation (7) may be written as

$$
\dot{\epsilon}=\dot{\lambda} \frac{\partial f\left(S, \vartheta^{*}\right)}{\partial S}, \quad-\dot{\vartheta}=\dot{\lambda} \frac{\partial f\left(S, \vartheta^{*}\right)}{\partial \vartheta^{*}} \quad \text { in } \Omega .
$$

The subdifferential constitutive relation (6) is very important in the theory of plasticity. Now we shall give some more detailed explanation. In fact, according to the theory of convex analysis, for any given $\left(S, \vartheta^{*}\right) \in \boldsymbol{\Sigma} \times \boldsymbol{\Theta}^{*}$, the subdifferential relation (6) is equivalent to the following inequality:

$$
\langle\dot{\epsilon}, S-T\rangle+\left\langle-\dot{\vartheta}, \vartheta^{*}-\phi^{*}\right\rangle \geq W^{*}\left(S, \vartheta^{*}\right)-W^{*}\left(T, \phi^{*}\right) \quad \forall\left(T, \phi^{*}\right) \in \mathbf{\Sigma} \times \mathbf{\Theta}^{*} .
$$

If $\dot{\epsilon} \neq 0$ and $\dot{\vartheta} \neq 0$, then $\left(S, \vartheta^{*}\right)$ must be on the yield surface, i.e., $f\left(S, \vartheta^{*}\right)=0$, and if $\left(T, \phi^{*}\right) \in \mathbf{K}$, the inequality (12) will degenerate to

$$
\langle\dot{\epsilon}, S-T\rangle+\left\langle-\dot{\vartheta}, \vartheta^{*}-\phi^{*}\right\rangle \geq 0 \quad \forall\left(T, \phi^{*}\right) \in \mathbf{K} .
$$

This is the general form of Drucker's postulate for a hardening material. We should stress here that in Drucker's postulate, the field variables $\left(T, \phi^{*}\right)$ should satisfy the yield condition, i.e., $\left(T, \phi^{*}\right) \in \mathbf{K}$. However, this constraint is relaxed in the generalized Drucker's hypothesis (12).

For linear hardening material, we can assume that $F\left(\vartheta^{*}\right)=\vartheta^{*}=H \vartheta$, where $H$ is a positive tensor. Hence we have

$$
\dot{\vartheta}=H^{-1} \dot{\vartheta}^{*}
$$

It is assumed that the body is initially in a natural state. During the large deformation process, the body $\Omega$ is subjected to a quasi-static loading system: body forces $b(x)$ in $\Omega$, surface tractions $t=\nu_{c} \bar{t}(x)$ on the boundary $\Gamma_{t}$. Then we have the following abstract governing equations:

(1) Geometric Equation:

$$
A_{1}(u) \dot{u}-\dot{\epsilon}=0 \quad \text { in } \Omega, \quad u=0 \quad \text { on } \Gamma_{\nu},
$$

(2) Equilibrium Equation:

$$
A_{1}^{*}(u) S-b=0 \quad \text { in } \Omega, \quad A_{1}^{*}(u) S-\nu_{c} \bar{t}=0 \quad \text { on } \Gamma_{t},
$$

(3) Constitutive Equation:

$$
(\dot{\epsilon},-\dot{\vartheta}) \in \partial W^{*}\left(S, \vartheta^{*}\right), \quad \vartheta^{*}=H \vartheta \quad \text { in } \Omega .
$$

It is rather unlikely that for given unit loading systems $\bar{t}$ on $\Gamma_{t}$, an exact loading factor and a complete solution $\left(u, S, \vartheta^{*}\right)$ can be found, because of the presence of two 
sources of nonlinearity (geometric nonlinearity and constitutive nonlinearity). Therefore, some approximate technique for estimating $\nu_{c}$ would be useful for engineering applications.

3. Variational inequality. Let $\boldsymbol{\Sigma}_{a}$ be a time-independent statically admissible space:

$$
\begin{aligned}
\boldsymbol{\Sigma}_{a}:=\{ & \left(v, T, \phi^{*}\right) \in \mathbf{U} \times \mathbf{\Sigma} \times \mathbf{\Theta}^{*} \mid A_{1}^{*}(v) S-b=0 \text { in } \Omega, \\
& A_{1}^{*}(v) S-\nu \bar{t}=0 \text { on } \Gamma_{t}, \\
& \left.v=0 \text { or } T=0, \phi^{*}=0 \text { in } \Omega \text { at the initial state }\right\},
\end{aligned}
$$

in which $\nu>0$ is a statically admissible load factor associated with $(v, S) \in \boldsymbol{\Sigma}_{a}$. Introducing the so-called gap function [4] $G: \mathbf{U} \times \boldsymbol{\Sigma} \rightarrow \tilde{\mathbf{R}}$ :

$$
G(v, T):=\left\langle-A_{2}(v) v, T\right\rangle_{\Omega}=\int_{\Omega} \frac{1}{2}(\nabla v)(v \nabla) T d \Omega,
$$

where $A_{2}$ is a geometric mapping: $A_{2}(v) v:=-\frac{1}{2}(\nabla v)(v \nabla)$; we may propose the following bounding theorem.

THEOREM 1. For any given time-independent statically admissible fields $\left(v, T, \phi^{*}\right) \in$ $\Sigma_{a}$, if the gap function satisfies $G(v, T) \geq 0$, then the following variational inequality holds:

$$
\nu_{c} \geq \nu(v, T)-\int_{\Omega} W^{*}\left(T, \phi^{*}\right) d \Omega .
$$

Proof. From the subdifferential including (6), we have

$$
W^{*}\left(T, \phi^{*}\right)-W^{*}\left(S, \vartheta^{*}\right) \geq\langle\dot{\epsilon}, T-S\rangle-\left\langle\dot{\vartheta}, \phi^{*}-\vartheta^{*}\right\rangle \quad \forall\left(T, \phi^{*}\right) \in \mathbf{\Sigma} \times \mathbf{\Theta}^{*} .
$$

Letting $u=v+\delta u$ and $\vartheta^{*}=\phi^{*}+\delta \vartheta^{*}$, and noting that $v, T$, and $\phi^{*}$ are timeindependent, we have $\dot{u}=\delta \dot{u}, \dot{\theta}^{*}=\delta \dot{\theta}^{*}$, and

$$
\dot{\epsilon}(u)=\dot{\epsilon}(v+\delta u)=A_{1}(v) \delta \dot{u}-2 A_{2}(\delta u) \delta \dot{u} .
$$

Substituting into (18) yields

$$
\begin{aligned}
W^{*}\left(T, \phi^{*}\right)-W^{*}\left(S, \vartheta^{*}\right) \geq & \left\langle A_{1}(v) \delta \dot{u}, T\right\rangle-\left\langle 2 A_{2}(\delta u) \delta \dot{u}, T\right\rangle \\
& -\left\langle A_{1}(u) \dot{u}, S\right\rangle+\left\langle H^{-1} \delta \vartheta^{*}, \delta \dot{\vartheta}^{*}\right\rangle \quad \forall\left(v, T, \phi^{*}\right) \in \mathbf{U} \times \mathbf{\Sigma} \times \boldsymbol{\Theta}^{*} .
\end{aligned}
$$

Integrating this inequality, and using Gaussian transformation, we obtain

$$
\begin{aligned}
\int_{\Omega} W^{*} & \left(T, \phi^{*}\right) d \Omega-\int_{\Omega} W^{*}\left(S, \vartheta^{*}\right) d \Omega \\
\geq & \left\langle\delta \dot{u}, A_{1}^{*}(v) T\right\rangle_{\bar{\Omega}}-\left\langle 2 A_{2}(\delta u) \delta \dot{u}, T\right\rangle_{\Omega}-\left\langle\dot{u}, A_{1}^{*}(u) S\right\rangle_{\bar{\Omega}} \\
& +\left\langle H^{-1} \delta \vartheta^{*}, \delta \dot{\vartheta}^{*}\right\rangle_{\Omega} \quad \forall\left(v, T, \phi^{*}\right) \in \mathbf{U} \times \mathbf{\Sigma} \times \boldsymbol{\Theta}^{*} \\
= & \langle\dot{u}, \nu(v, T) \bar{t}\rangle_{\Gamma_{1}}-\left\langle 2 A_{2}(\delta u) \delta \dot{u}, T\right\rangle_{\Omega}-\left\langle\dot{u}, \nu_{c} \bar{t}\right\rangle_{\Gamma_{t}} \\
& +\left\langle H^{-1} \delta \vartheta^{*}, \delta \dot{\vartheta}^{*}\right\rangle_{\Omega} \quad \forall\left(v, T, \phi^{*}\right) \in \mathbf{\Sigma}_{a} .
\end{aligned}
$$


By performing time-integration and considering the property of the super-potential $W^{*}$, we have

$$
\begin{aligned}
\int_{\Omega} W^{*}\left(T, \phi^{*}\right) d \Omega-\int_{\Omega} W^{*}\left(S, \vartheta^{*}\right) d \Omega & \\
\geq & \langle u, \nu(v, T) \bar{t}\rangle_{\Gamma_{1}} l_{0}^{t}-\left.\left\langle A_{2}(\delta u) \delta u, T\right\rangle_{\Omega}\right|_{0} ^{t}-\left.\left\langle u, \nu_{c} \bar{t}\right\rangle_{\Gamma_{l}}\right|_{0} ^{t}+\left.\frac{1}{2}\left\langle\delta \vartheta^{*}, H^{-1} \delta \vartheta^{*}\right\rangle\right|_{0} ^{t} \\
=\left(\nu(v, T)-\nu_{c}\right)\langle u, \bar{t}\rangle_{\Gamma_{1}}+G((u-v), T)+\frac{1}{2}\left\langle\left(\phi^{*}-\vartheta^{*}\right), H^{-1}\left(\phi^{*}-\vartheta^{*}\right)\right\rangle_{\Omega} & \forall\left(v, T, \phi^{*}\right) \in \Sigma_{a} .
\end{aligned}
$$

For any given $(v, T) \in \Sigma_{a}$, if $G(v, T) \geq 0$, we have $G(u-v, T) \geq 0$. Removing the positive terms on the right-hand side of (21), noticing that $\langle u, \bar{t}\rangle_{\Gamma_{1}}>0$ and using the property of the superpotential, the variational inequality (17) is proved. Q.E.D.

4. Application. Consider a simply supported beam fully restrained from axial motion and subjected to a uniformly distributed load. For the sake of simplicity, the axial component of the displacement is neglected. The geometrical parameters involved and the coordinate systems are defined in Fig. 1. The abstract governing equations in this case have the form:

$$
\left\{\begin{array}{l}
M^{\prime \prime}+\left(N w^{\prime}\right)^{\prime}+\nu p=0 \\
N^{\prime}=0 \\
\epsilon=\frac{1}{2} w^{\prime 2} \\
\kappa=-w^{\prime \prime}
\end{array}\right.
$$

where $w$ is displacement in the $y$ direction, and $M$ and $N$ denote bending moment and axial force, respectively. Corresponding generalized strains are $(\kappa, \epsilon)$. For a rectangular cross section of the beam, the yield function is described by $[6,8]$ :

$$
f\left(M, N, \theta^{*}\right)=\frac{|M|}{M_{s}\left(\theta^{*}\right)}+\left(\frac{N}{N_{s}\left(\theta^{*}\right)}\right)^{2}-1,
$$

in which $M_{s}=\sigma_{s} h^{2} / 4, N_{s}=\sigma_{s} h$, and $\sigma_{s}=\sigma_{b}+\theta^{*}=\sigma_{b}(1+\bar{H} \theta),\left(\bar{H}=H / \sigma_{b}\right)$. According to the general definition, given Eq. (7), the internal parameter $\vartheta$ in the case of a beam-string is

$$
\vartheta=\frac{1}{L N_{s}} \int_{0}^{t} d t \int_{0}^{L}(M \dot{\kappa}+N \dot{\epsilon}) d x .
$$

It was shown in Ref. [11] that when $\delta \geq h / 2$, the stress profile on the yield condition is reduced to the point $M=0, N=N_{s}$. Introducing these values into Eq. (24), the time integration can be readily performed to give

$$
\vartheta=\frac{1}{L} \int_{0}^{L} \epsilon d x
$$

If small deflections are considered, $\delta<h / 2$, the definition of the parameter $\vartheta$ should include both $\kappa$ and $\epsilon$, but our interest is in the large deflection range. 


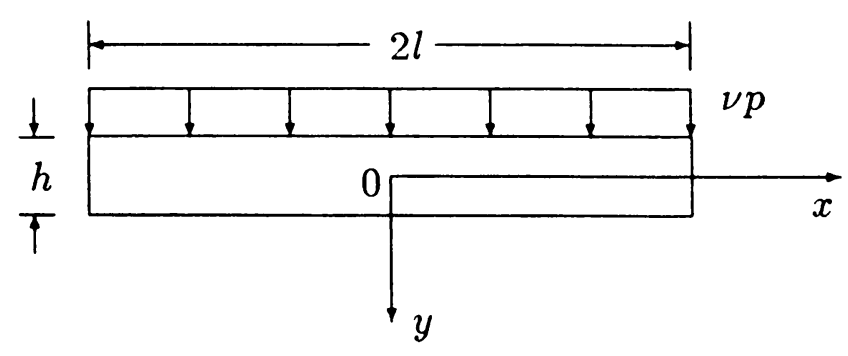

FIG. 1. Beam under a uniform loading.

The gap function (14) in this case has the form

$$
G(w, N)=\int_{0}^{l} \frac{1}{2} N w^{2} d x
$$

From the loading conditions, it is easy to see that $N \geq 0$, which implies that $G(w, N) \geq 0$. Hence for given statically admissible fields $(w, M, N)$, we have the following inequality:

$$
\nu_{c} \geq \nu(w, M, N)-\int_{0}^{l} W^{*}\left(M, N, \theta^{*}\right) d x .
$$

Now, let us choose the following statically admissible fields:

$$
w=w_{0}\left(1-\frac{x^{2}}{l^{2}}\right), \quad M=\eta M_{b}\left(1-\frac{x^{2}}{l^{2}}\right), \quad N=\mu \delta N_{b},
$$

where $\eta, \mu \geq 0$ are unknown parameters, $M_{b}=\sigma_{b} h^{2} / 4, N_{b}=\sigma_{b} h$, and $\delta=w_{0} / h$. Obviously, at the initial state $(\delta=0), w(\delta)=\theta^{*}(\delta)=0$. Substituting (28) into (22) and (23) and letting $\theta^{*}=H \theta(\delta)$, we get

$$
\begin{gathered}
\nu(\delta, \eta, \mu)=\eta+4 \mu \delta^{2}, \quad p=\frac{2 M_{b}}{l^{2}}, \\
f(\dot{\eta}, \mu, \delta)=\frac{\eta\left(1-x^{2} / l^{2}\right)}{1+\bar{H} \theta(\delta)}+\frac{\mu^{2} \delta^{2}}{(1+\bar{H} \theta(\delta))^{2}}-1 .
\end{gathered}
$$

Using Eqs. (22) and (25), the internal variable parameter $\vartheta$ can be expressed in terms of $\delta$,

$$
\vartheta=\frac{2}{3} c^{2} \delta^{2} \quad(c:=h / L)
$$

Let

$$
\nu^{-}(\eta, \mu, \delta)=\nu(\delta, \eta, \mu)-\int_{0}^{l} W^{*}\left(M(\eta), N(\mu, \delta), \theta^{*}(\delta)\right) d x
$$

Then for some given $\delta \geq 0$, we have the optimization lower bound problem

$$
\max _{\eta \geq 0} \max _{\mu \geq 0} \nu^{-}(\eta, \mu, \delta) \text {. }
$$

According to the property of the superpotential (8), we can give a penalty type construction of $W^{*}[3,9,10]$ :

$$
W_{p}^{*}(\eta, \mu, \delta, \alpha)=\frac{f(\eta, \mu, \delta)}{2 \alpha} \max \{0, f\},
$$


where $\alpha>0$ is a penalty factor. It is easy to prove that for any given $\eta, \mu, \delta \geq 0$, we have

$$
W^{*}\left(M(\eta), N(\mu, \delta), \theta^{*}(\delta)\right)=\lim _{\alpha \rightarrow 0^{+}} W_{p}^{*}(\eta, \mu, \delta, \alpha) .
$$

So for any given strictly decreasing sequence $\left\{\alpha_{n} \geq 0(n=1,2, \ldots)\right\}$, the limit of the following unconstrained optimization problem,

$$
\nu_{0}(\delta)=\lim _{n \rightarrow+\infty} \max _{\eta \geq 0} \max _{\mu \geq 0}\left\{\eta+4 \mu \delta^{2}-W_{p}^{*}\left(\eta, \mu, \delta, \alpha_{n}\right)\right\},
$$

gives the optimal lower bound of the safety factor $\nu_{c}$, i.e., $\mu_{0}(\delta) \leq \nu_{c}(\delta)$.

5. Results and discussion. In order to illustrate the optimization procedure, calculations were run for a particular beam with the slenderness parameter equal to $c=h / L=0.2$. The results are shown in Table 1 for a limiting case of a rigid, perfectly plastic material and also in Fig. 2 for a linear work-hardening material with two different values of the hardening parameter $\bar{H}$.

TABLE 1. Numerical results for large deformation plastic beam

$$
\text { (c }=.2, \bar{H}=0) \text {. }
$$

\begin{tabular}{|c|c|c|c|c|c|c|}
\hline$\delta$ & 0.25 & 0.50 & 0.75 & 1.00 & 1.50 & 2.00 \\
\hline \hline$\eta$ & .781 & .340 & .289 & .10 & .03 & .02 \\
\hline$\mu$ & .468 & .810 & .844 & .948 & .980 & .490 \\
\hline$\nu_{0}$ & 1.25 & 1.97 & 2.82 & 3.89 & 5.94 & 7.93 \\
\hline Membrane solution & 1.0 & 2.0 & 3.0 & 4.0 & 6.0 & 8.0 \\
\hline
\end{tabular}

For comparison, shown in Fig. 2 (dash line) and Table 1 (last row) is a purely membrane solution obtained from the set of equations (22) by assuming $M=\kappa=$ 0 and $N=N_{b}$. Using the dimensional quantities defined earlier, this solution is represented by

$$
\nu_{c}=4 \delta \text {. }
$$

It can be seen that for large $\delta$ the numerical solution approaches the analytical one, as expected. An interesting property of the present analysis is revealed by examining the variation of the parameters $\eta(\delta)$ and $\mu(\delta)$ with the dimensional central deflection $\delta$, shown in Table 1 . The bending moment starts at $M=M_{b}$ and is rapidly diminishing reaching practically zero for deflection equal to the beam thickness. At the same time, the membrane force is steadily increasing towards the fully plastic axial force $N_{b}$. A transition from flexural to membrane response of plastic beams has been reported in earlier studies on this subject, for example in Ref. [11].

The effect of strain hardening on the force deflection diagram is clearly seen in Fig. 2. The present results are also fully compatible with previous solutions of the same problem, presented by Symonds and Jones [11, 12].

The main emphasis of the present note was to develop a new analytical tool for solving large deflection problems with strain-hardening effect. We think that the 


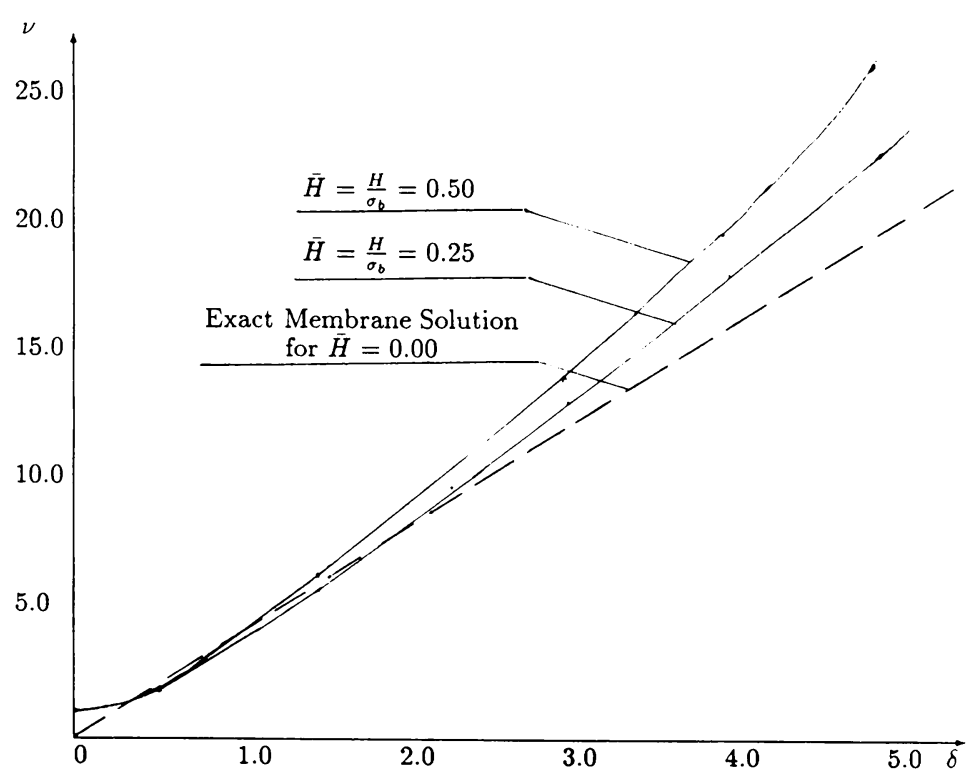

FIG. 2. Comparison of membrane solution for deflection with bounds for beam

present method, based on the construction of simple kinematically admissible fields and optimization techniques, may be useful in solving a wide range of engineering problems for shells.

6. Conclusions. In this paper, we have proved that in the case of geometrical nonlinear plastic analysis, the existence of lower bounds for load intensity is directly related to the properties of the gap function. Based on the theory of convex analysis, a new lower bound theorem for the load intensity is established for materials with strain-hardening effects. By introducing the concept of the complementary plastic superpotential, the yield condition is relaxed, which is of great consequence in deriving approximate solutions to engineering problems. A penalty type variational approximation is suggested to solve the illustrative problem. The numerical results show that the new lower bound theorem works very well.

\section{REFERENCES}

[1] A. M. Arthurs, Complementary Variational Principles, Second edition, Clarendon Press, 1980

[2] J. T. Oden and J. N. Raddy, On the dual complementary variational principles in mathematical physics, Int. J. Eng. Sci. 12, 1-29 (1974)

[3] Yang Gao, On the Complementary Principles in Elastoplastic Systems and Panpenalty Finite Element Methods, Ph.D. Thesis, Tsinghua University, Beijing, 1985

[4] Yang Gao and G. Strang, Geometrical nonlinearity: potential energy, complementary energy and the gap function, Quart. Appl. Math. 47, 487-504 (1989)

[5] E. T. Onat, The influence of geometry changes on the load-deformation behavior of plastic solids, in Plasticity, Proc. 2nd U. S. Naval Struc. Mech., Pergamon Press, 225-238, 1960 
[6] J. Ploch and T. Wierzbicki, Bounds for large plastic deformations of dynamically loaded continua and structure.s, Internat. J. Solids and Structures, 1980

[7] I. Ekeland and R. Temam, Convex Analysis and Variational Problems, North-Holland, 1976

[8] T. Wierzbicki, Berechnung Dynamisch Beanspruchter Konstruktionen, Werner-Verlag, 1983

[9] Yang Gao, Panpenalty finite element programming for limit analysis, to be published in Computer and Structure, 1988

[10] Yang Gao, On the complementary bounding theorems of limit analysis, to appear in Internat. J. Solids and Structures, 1988

[11] P. S. Symonds and N. Jones, Impulsive loading of fully clamped beams with finite deflections and strain-rate sensitivity, Internat. J. Mech. Sci. 14, 49-69 (1972)

[12] N. Jones, Finite deflections of a rigid-viscoplastic strain-hardening annular plate loaded impulsively, J. Appl. Mech. 35, 349-356 (1968)

[13] J. J. Moreau, On unilateral constraints, friction and plasticity, in New Variational Techniques in Mathematical Physics, C.I.M.E. Edizioni Gremonese, Roma, 1974 